\title{
n-3 Polyunsaturated Fatty Acids: Promising Nutrients for Preventing Cardiovascular Disease
}

\author{
Shusuke Yagi 1, 2, 6 , Daiju Fukuda ${ }^{1,5}$, Ken-ichi Aihara ${ }^{3}$, Masashi Akaike ${ }^{4}$, Michio Shimabukuro ${ }^{5,7}$ and \\ Masataka Sata ${ }^{1}$ \\ ${ }^{1}$ Department of Cardiovascular Medicine, Tokushima University Graduate School of Biomedical Sciences, Tokushima, Japan \\ ${ }^{2}$ Department of Community Medicine and Human Resource Development, Tokushima University Graduate School of Biomedical \\ Sciences, Tokushima, Japan \\ ${ }^{3}$ Department of Community Medicine for Diabetes and Metabolic Disorders, Tokushima University Graduate School of Biomedical \\ Sciences, Tokushima, Japan \\ ${ }^{4}$ Department of Medical Education, Tokushima University Graduate School of Biomedical Sciences, Tokushima, Japan \\ ${ }^{5}$ Department of Cardio-Diabetes Medicine, Tokushima University Graduate School of Biomedical Sciences, Tokushima, Japan \\ ${ }^{6}$ Department of Internal Medicine, Shikoku Central Hospital, Ehime, Japan \\ ${ }^{7}$ Department of Diabetes, Endocrinology and Metabolism, School of Medicine, Fukushima Medical University, Japan
}

The adoption of the Western-style diet, with decreased fish intake and lack of exercise, has increased the prevalence of cardiovascular disease (CVD) in Japan. Statin treatment has been established to reduce the risk of cardiovascular events; however, $60 \%-70 \%$ of these events occur despite its use. Thus, the residual risk for CVD should be identified and resolved to reduce further cardiovascular events. The serum levels of $n-3$ polyunsaturated fatty acids (PUFAs), including eicosapentaenoic acid and docosahexaenoic acid, are reportedly associated with an increased incidence of cardiovascular events and mortality, whereas the addition of n-3 PUFA treatment to the statin treatment decreases cardiovascular events. Similar to statins, n-3 PUFAs have pleiotropic effects in addition to lipid-modifying effects. Pre-clinical and clinical studies have shown that n-3 PUFAs prevent cardiovascular events by ameliorating endothelial function and attenuating lipid accumulation, vascular inflammation, and macrophage recruitment, thereby causing coronary plaque development and rupture. Taken together, n-3 PUFAs are comprehensively able to attenuate the atherogenic response. Therefore, $n-3$ PUFA intake is recommended to prevent cardiovascular events, particularly in patients with multiple cardiovascular risk factors.

Key words: Eicosapentaenoic acid, Docosahexaenoic acid, Cardiovascular disease, Residual risk

This article is distributed under the terms of the latest version of CC BY-NC-SA defined by the Creative Commons Attribution License.

\section{Introduction}

The wide adoption of Western-style diet, with decreased fish intake and lack of exercise, in the motorized society has increased the prevalence of cardiovascular disease (CVD) in Japan. Therefore, comprehen-

Address for correspondence: Shusuke Yagi, Department of Cardiovascular Medicine/ Department of Community Medicine and Human Resource Development, Tokushima University Graduate School of Biomedical Sciences, Tokushima, Japan, 3-18-15 Kuramoto-cho, Tokushima-city, Tokushima 770-8503, Japan

E-mail: syagi@tokushima-u.ac.jp

Received: July 13, 2017

Accepted for publication: July 19, 2017 sive management and education regarding diet and exercise are essential to prevent CVD and to manage conventional CVD risk factors such as hypertension, diabetes, dyslipidemia, and smoking. Statin treatment of dyslipidemia has been established by a larger body of evidence; however, the reduction of cardiovascular events is $30 \%-40 \%$ in patients for the primary and secondary prevention of cardiovascular events, indicating that statin use continues to cause $60 \%-70 \%$ of cardiovascular events ${ }^{1}$. Therefore, the residual risk for CVD should be identified and resolved to reduce further cardiovascular events in this era of statins.

Decreased serum levels of $n-3$ polyunsaturated fatty acids (PUFAs), including eicosapentaenoic acid (EPA) and docosahexaenoic acid (DHA), are report- 

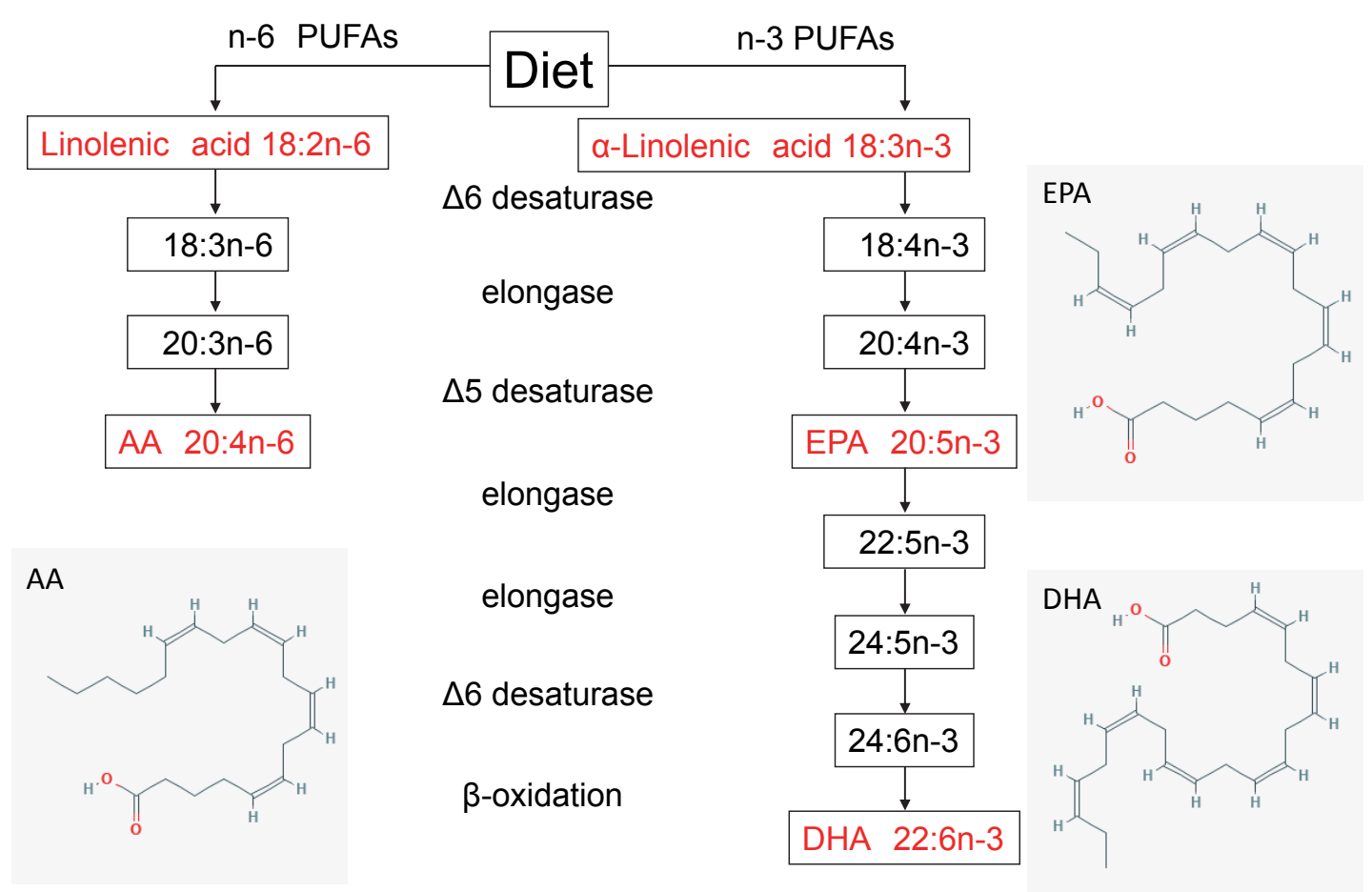

Fig. 1. The metabolism of PUFAs. AA, arachidonic acids; EPA, eicosapentaenoic acid; DHA, docosahexaenoic acid (https://pubchem.ncbi.nlm.nih.gov/compound) ${ }^{15)}$

edly associated with an increased incidence of cardiovascular events and mortality ${ }^{2)}$, whereas n-3 PUFA treatment add-on to the statin treatment decreases cardiovascular events in Japan ${ }^{3)}$. Therefore, the decreased level of n-3 PUFAs appears to be a residual therapeutic target to overcome cardiovascular events, particularly in patients who do not regularly ingest fish, which is a source of n-3 PUFAs. In this review, we focus on the potential and mechanisms of $n-3$ PUFAs as promising nutrients against the residual risk for CVD.

\section{Metabolism of n-3 PUFAs}

n-3 PUFAs are derived from fish and fish oil. Once consumed in the diet, n-3 PUFAs are absorbed from the gastrointestinal tract and transported to the liver as triglycerides via chylomicron particles. Upon being transported to the liver, n-3 PUFAs are used as a source of triglycerides in lipoprotein particles including low-density lipoprotein (LDL). Triglycerides mainly comprises oleic acids and saturated fatty acids, and phospholipids mainly comprises PUFAs. n-3 PUFAs are released into the blood as plasma phospholipids from the liver and incorporated into cell membrane phospholipids throughout the body; some are stored in the adipose tissue as triglycerides. Therefore, increas- ing one's consumption of fish oil increases n-3 PUFA concentrations within the plasma lipids and organs.

n-3 PUFAs are derived from $\alpha$-linolenic acid (ALA), whereas n-6 PUFAs are derived from linolenic acid. Both PUFAs are metabolized by desaturase and elongase (Fig. 1). Linolenic acid is metabolized to arachidonic acid (AA), which is a precursor of prostaglandins, thromboxanes, and leukotrienes ${ }^{4}$. ALA is metabolized to EPA and DHA, which are precursors of prostaglandins and leukotrienes. Goyons et al. showed that nearly $7 \%$ of dietary ALA was incorporated into EPA, and only $0.013 \%$ of ALA was converted to DHA through hepatic conversion with the tracer model, which was developed based on the averaged ${ }^{13} \mathrm{C}$ data of healthy subjects ${ }^{5)}$. Hussein et al. showed that $0.3 \%$ and $<0.01 \%$ of ALA is converted to EPA and DHA, respectively, in patients with hyperlipidemia ${ }^{6}$. The biochemical and clinical significance of the retro conversion of DHA to EPA is unknown ${ }^{4)}$. Although n-3 PUFAs are essential for a healthy life, particularly for normal growth and development ${ }^{7}$, only small amounts of ALA can be converted to EPA or DHA. Thus, n-3 PUFAs are called "essential fatty acids" and must be ingested as a part of the $\operatorname{diet}^{8)}$. 


\section{Statins Prevent CVD by Attenuating Atherogenic Steps}

The concept that atherosclerosis results from vascular inflammation is widely recognized. The accumulation of CVD risk factors provokes vascular inflammation and increases the atherosclerotic burden in the coronary and other arteries, resulting in cardiovascular events such as acute coronary syndrome (ACS). Atherogenic vascular inflammation comprises the following: 1) endothelial dysfunction; 2) lipid accumulation; $3)$ vascular inflammation and recruitment of macrophages; 4) plaque development through the proliferation and migration of smooth muscle cells (SMCs); and 5) plaque vulnerability leading to plaque rupture9).

3-Hydroxy-3-methylglutaryl coenzyme A reductase inhibitors, called statins, inhibit the key enzyme in cholesterol biosynthesis and have been established to reduce cardiovascular events and all-cause mortality rates. Statins reduce the intracellular cholesterol synthesis and upregulate the LDL receptors in the liver, leading to reductions in the circulating levels of LDL cholesterol by $20 \%-60 \%{ }^{10,11)}$. Furthermore, statins have both cholesterol-lowering and pleiotropic effects on the cardiovascular system, including anti-inflammatory, antioxidant, and improved nitric oxide bioavailability ${ }^{12-14}$. Statins can attenuate all the above features of atherogenesis. However, the ability of statins to reduce cardiovascular events has room for improvement, and the residual risk for CVD should be identified.

\section{Statins Decrease n-3 PUFA Levels}

Statin and diet therapy reportedly modulates n-3 PUFA composition. Jula et al. reported that, compared with placebo, simvastatin treatment significantly reduced DHA, but not EPA levels, in patients with hyperlipidemia ${ }^{15)}$. Nozue et al. reported that pitavastatin decreased the serum DHA/AA ratio, but not the EPA/AA ratio, in patients with $\mathrm{CVD}^{16)}$. Kuris et al. showed that strong statins, including atorvastatin, rosuvastatin, and pitavastatin, reduced the serum levels of EPA and DHA in proportion to decreases in LDL cholesterol in patients with $\mathrm{CVD}^{17)}$. Harris et al. reported that simvastatin increased the AA/EPA and AA/DHA ratios ${ }^{18)}$. Nakamura et al. reported that pravastatin and simvastatin increased serum AA levels but did not affect serum EPA levels, which resulted in a decreased EPA/AA ratio ${ }^{19)}$.

The mechanisms by which statin treatment reduces EPA/AA or DHA/AA ratio or EPA and DHA levels have not been completely elucidated, but it is speculated that statin and diet therapy modulates the enzyme activity of PUFA synthesis, including desaturase and elongase (Fig. 1). Thus, patients who take statins may be recommended to take greater amounts of n-3 PUFAs to prevent cardiovascular events.

\section{Low Serum n-3 PUFA Level is a Risk Factor for CVD}

A reduced serum n-3 PUFA level is associated with an increased risk of cardiovascular events. Epidemiologic studies conducted on Greenland Inuit have shown a connection between a high seafood intake containing high n-3 PUFA levels and a low cardiovascular morbidity ${ }^{20}$. In Japan, atherosclerotic lesions, evaluated by pulse wave velocity of the aorta and intima-media thickness of the carotid artery evaluated by ultrasonography are lower in both men and women in fishing villages than in farming villages ${ }^{21)}$. The Japan Public Health Center-based study showed that, compared with a modest fish intake, a higher fish intake was associated with a substantially reduced risk of coronary heart disease, primarily nonfatal cardiac events, among middle-aged individuals ${ }^{22)}$. In the Netherlands, mortality rates from CVD were $>50 \%$ lower in patients who consumed at least $30 \mathrm{~g} /$ day of fish than in those who did not consume fish, suggesting that the consumption of one or two fish dishes per week prevents $\mathrm{CVD}^{23)}$. A meta-analysis performed by Wang et al. showed that an increased consumption of n-3 PUFAs from fish or fish oil supplements, but not of ALA, reduces all-cause mortality, cardiac death, and sudden death rates ${ }^{24)}$. He et al. showed an inverse association between n-3 PUFA intake and the prevalence of $\mathrm{CVD}^{25}$. These epidemiologic population studies showed that a reduced n-3 PUFA intake is a risk factor for cardiovascular events.

\section{Randomized Controlled Trials Show that $\mathrm{n}-3$ PUFAs Prevent Cardiovascular Events}

For the primary prevention of CVD, the Japan EPA Lipid Intervention Study (JELIS), a randomized trial of 18,645 patients with hypercholesterolemia showed a $19 \%$ reduction in major cardiovascular events in patients randomized to a statin + highly purified EPA $1800 \mathrm{mg} /$ day compared with those randomized to a statin alone ${ }^{3)}$. For the secondary prevention of CVD, the DART study, a randomized controlled trial performed in the United Kingdom, showed that patients who recovered from myocardial infarction and advised to eat fatty fish had a $29 \%$ reduction in the 2-year all-cause mortality compared with those who were advised to reduce their fat intake and increase their cereal fiber intake ${ }^{26}$. GISSI-Prevenzi- 


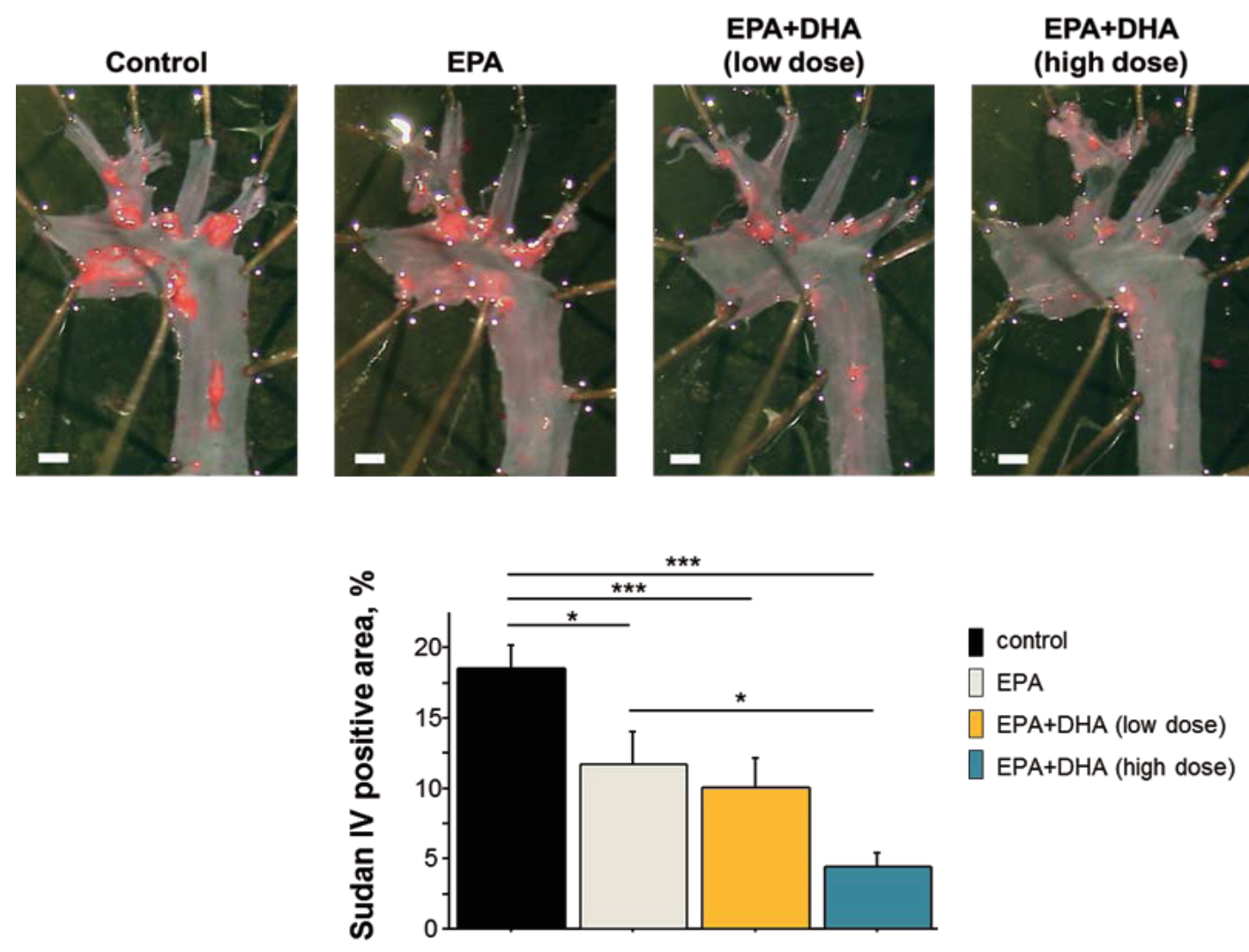

Fig. 2. The effects of n-3 PUFAs on the progression of atherosclerosis in Apoe ${ }^{-1-}$ mice. En face Sudan IV staining of the aorta. The high-dose EPA + DHA group, which received an additional dose of DHA to the same dose of EPA as that in the EPA group, demonstrated a greater reduction in atherogenesis compared with the EPA group. The low-dose EPA+DHA group, in which half the amount of EPA in the EPA group was replaced with a similar amount of DHA, demonstrated similar suppression of atherogenesis as that in the EPA group ${ }^{30)}$.

one, a randomized controlled trial performed in Italy, showed that an intake of $\mathrm{n}-3$ PUFAs (1 g daily), but not of vitamin E (300 mg daily), showed a significant reduction in the total and cardiovascular mortality as well as in the sudden cardiac death in patients with post-myocardial infarction ${ }^{27)}$. A meta-analysis of randomized controlled trials of a lipid-lowering intervention with placebo or a usual diet concerning mortality showed that statins and n-3 PUFAs are the most favorable lipid-lowering interventions with reduced risks of the total and cardiovascular mortality ${ }^{28)}$. Thus, these clinical trials indicated that n-3 PUFA intake could decrease cardiovascular events.

\section{Differential Effects of DHA and EPA}

Clinical studies have shown the effects of fish oil and n-3 PUFAs on cardiovascular events; however, the differential effects of DHA and EPA have been less well established. It has been reported that a low level of DHA is associated with all-cause death, which was not observed in patients with a low level of EPA after acute myocardial infarction ${ }^{2)}$, and that higher plasma DHA, but not EPA, is associated with the reduced progression of coronary atherosclerosis in women with coronary artery disease $(\mathrm{CAD})^{29)}$. These results suggest that DHA exerts a protective effect on CAD and indicates the possibility that the administration of DHA can reduce cardiovascular events.

We showed that the combination of EPA and DHA has additional anti-inflammatory and anti-atherosclerotic effects with Western-type diet-fed Apoe ${ }^{-/-}$ mice (Fig. 2) ${ }^{30}$. In this study, high-dose EPA + DHA more effectively reduced atherogenesis than did EPA alone, in which the amount of EPA in these two treatments was similar. In addition, low-dose EPA + DHA, in which half the amount of EPA in the EPA group was replaced with a similar amount of DHA, reduced atherogenesis to the same level as that in the EPA group. The results indicated that DHA had additional anti-atherosclerotic effects when combined with EPA. DHA has a nature of better tissue distribution than 
EPA $^{31)}$. The bioavailability of DHA may explain the preferable effects of DHA on anti-atherosclerogenesis. Large clinical trials have shown the evidence of the effects of EPA or fish oil on the decrease in cardiovascular events; however, there has been no large clinical trial to show the preferable effects of DHA on cardiovascular events. Thus, large, clinical cohort studies are needed to clarify the differential effects of EPA and DHA on preventing cardiovascular events.

\section{Mechanisms by which n-3 PUFAs Prevent CVD: Attenuating All Atherogenic Features}

\section{1) Endothelial Dysfunction}

Endothelial dysfunction caused by a loss of endothelium-derived nitric oxide initiates atherosclerotic formation ${ }^{32}$. The accumulation of lifestyle-related diseases triggers endothelial damage at the sites of disturbed laminar flow, such as branches, bifurcations, and curvatures, which lead to the breakdown of endothelial continuity ${ }^{9)}$.

A meta-analysis of 16 randomized placebo-controlled trials including 901 participants showed that n-3 PUFA intake increased the flow-mediated vasodilation, which is a noninvasive measure of nitric oxide dependent on endothelial function, by $2.30 \%(P=0.001)$ at a dose of $0.45-4.5 \mathrm{~g} /$ day over a median 56 days compared with placebo ${ }^{33)}$. Our observational study showed that serum DHA levels, but not EPA levels, in patients with CAD is associated with endothelial function when evaluated by flow-mediated vasodilatation, suggesting that a low serum DHA level is a risk factor for endothelial dysfunction, leading to $\mathrm{CVD}^{34)}$. A double-blind placebo-controlled trial showed that DHA, but not EPA, enhances vasodilation in response to acetylcholine, which induces endogenous nitric oxide release ${ }^{35,36}$.

It was reported that n-3 PUFAs enhance endothelial nitric oxide synthase (eNOS) expression and activation, leading to vasodilation ${ }^{37,38)} \cdot \mathrm{n}-3$ PUFAs induce the translocation of eNOS from caveolin in the cell membrane to the cytosol, leading to eNOS system enhancement and activation, which may be a reason for n-3 PUFA-induced vasodilation ${ }^{39)}$. Another study showed that vascular endothelial dysfunction induced by palmitic acid, which is a saturated fatty acid, is attenuated by EPA treatment by the inhibition of longchain acyl-CoA synthase expression ${ }^{40)}$. Thus, endothelial dysfunction may be ameliorated by n-3 PUFAs via eNOS-dependent or eNOS-independent mechanisms.

\section{2) Lipid Accumulation}

Endothelial damage evokes the subendothelial retention of cholesterol-containing plasma lipoproteins in coronary arteries. LDL and triglyceride-rich lipo- proteins (remnant cholesterol) enter the subendothelial space via the damaged endothelial layer. LDL receives oxidative modifications to oxidized LDL, and small-dense LDL is more easily oxidized. Subsequently, oxidized LDL and triglyceride-rich lipoproteins could be taken up by macrophages for clearance, leading to vascular inflammation ${ }^{41)}$.

n-3 PUFAs reduce plasma triglycerides by approximately $20 \%-30 \%$ but have minimal effects on total, high-density lipoprotein, and LDL cholesterols ${ }^{42)}$. A meta-analysis of 17 large population-based studies showed that a high triglyceride level is an independent risk factor for $\mathrm{CVD}^{43)}$. However, compared with decreases in LDL cholesterol, insufficient data are available to document CVD benefits from decreases in plasma triglyceride in randomized placebo-controlled trials ${ }^{44)}$.

EPA reportedly reduces serum small-dense LDL and remnant lipoprotein particles in metabolic syndrome ${ }^{45)}$. The reduction of small-dense LDL by EPA treatment is due to the suppression of triglyceride production in the liver by EPA ${ }^{45)}$. Thus, n-3 PUFA treatment reduces the substrate of oxidative LDL or remnant cholesterol taken up by macrophages.

\section{3) Vascular Inflammation and Macrophage Recruit- ment}

Endothelial damage that occurs after lipid accumulation increases the adhesion of leukocytes to the endothelium by upregulating the expression of adhesion molecules, such as intercellular adhesion molecule 1 (ICAM-1), vascular cell adhesion molecule-1 (VCAM1), E-selectin, or P-selectin, and enhances their leukocyte permeability to sub-endothelium. Thus, adhesion molecules are critical to the initiation of vascular inflammation. The injured endothelium releases cytokines and growth factors, such as monocyte chemoattractant protein 1 (MCP-1) that act as chemotactic factors attracting monocytes and $\mathrm{T}$ cells to the vessel wall, resulting in vascular inflammation ${ }^{46}$. Inflammatory conditions recruit circulating monocytes and develop them into macrophages in the sub-endothelium.

Several randomized clinical trials have shown that n-3 PUFAs decrease the expression of cell adhesion molecules (e.g. VCAM-1, ICAM-1, and E-selectin) ${ }^{47}$. in vitro studies have shown that DHA, but not EPA, decreased the expression of cell adhesion molecules and monocyte adhesion to endothelial cells ${ }^{48)}$. n-3 PUFAs attenuated the expression of adhesion molecules on the surface of cultured human endothelial cells, monocytes, and lymphocytes ${ }^{48}$. The n-3 PUFA-induced attenuation of the expression of adhesion molecules was accompanied by a decreased binding of human lymphocytes and monocytes to cytokine-stimulated 
A

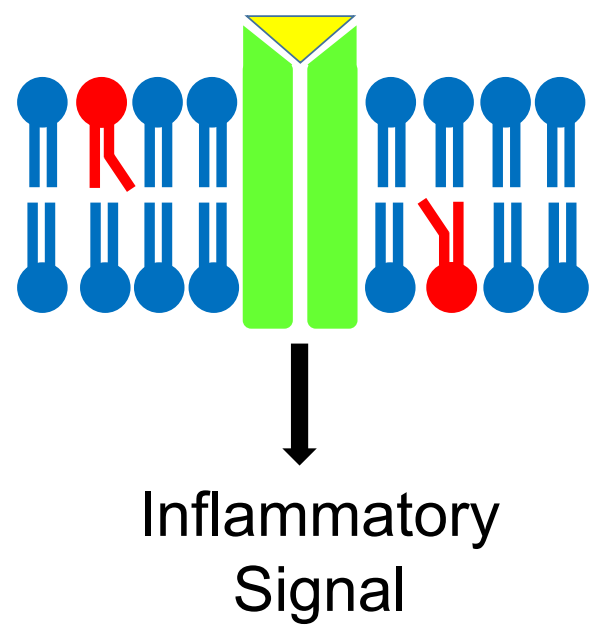

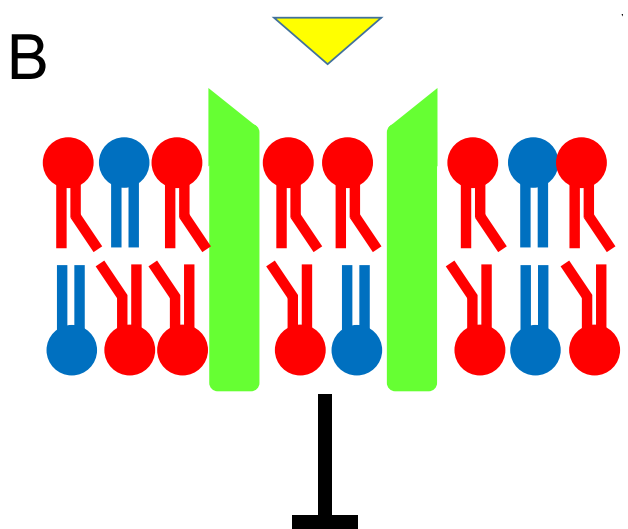

Inflammatory Signal

Saturated fatty acids

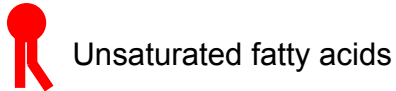

TLR4R

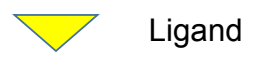

Fig. 3. The presumed mechanism of the anti-inflammatory effect of n-3 PUFAs. A: Unsaturated fatty acids-poor membrane B: Unsaturated fatty acids-rich membrane

endothelial cells ${ }^{48)}$. It has been shown that resolvins and $G$ protein-coupled receptor 120 , which are metabolites of EPA and DHA, play crucial roles in the active resolution of inflammation and are involved in the mechanisms of n-3 PUFA-induced anti-inflammatory effects. An ex vivo and animal study showed that $n-3$ PUFAs reduce the production of pro-inflammatory cytokines, including tumor necrosis factor (TNF)- $\alpha$, interleukin (IL)-1, and IL-6, following the lipopolysaccharide (LPS) stimulation of monocytes/lymphocytes ${ }^{46,49)}$. Clinical studies have shown that fish oil supplementation may inhibit the production of cytokines, including IL- $1 \beta$ and TNF- $\alpha^{50)}$.

The molecular mechanism of the anti-inflammatory effects of n-3 PUFAs has not been fully elucidated. The plasma membrane comprises a lipid raft, phospholipid bilayer with cholesterol microdomains, sphingolipids, and lipid-anchored proteins, and plays a crucial role in signal transduction as a signaling platform. Cell membranes with n-3 PUFA-rich phospholipids have an increased fluidity than those with $n-6$ PUFA-rich phospholipids.

Toll-like receptor (TLR) 4 is the receptor for LPS, which plays a critical role in the inflammatory signaling pathway, including the nuclear factor-kappa $\beta$ pathway. TLR4 stimulation induces the expression of pro-inflammatory cytokines and chemokines in various cell types, leading to the activation of NLR family pyrin domain containing (NLRP) 3 inflammasome when combined with the uptake or intracellular formation of cholesterol crystals ${ }^{51,52)}$. The most crucial TLR ligands are thought to be modified forms of LDL in atherosclerosis in addition to LPS. The initial step in signal transduction by TLR4 is the dimerization of two receptor chains induced by the binding of myeloid differentiation factor (MD)-2 to the lipid A moiety of LPS. Enhancing the dimerization of the cell membrane fluidity of the two receptor chains is important. We showed that n-3 PUFA treatment to the Westerntype diet-fed $A p o e^{-/-}$mice significantly decreased the progression of atherosclerotic lesions and TLR 4 expression in lipid rafts on macrophages ${ }^{30)}$. These results suggested that n-3 PUFAs modify TLR4 localization by attenuating TLR 4 dimerization on the cell membrane, resulting in inhibited TLR4 expression (Fig. 3) ${ }^{53)}$.

\section{4) Plaque Development through the Proliferation and Migration of SMCs}

The resulting increase in chemotactic factors stimulates the proliferation and migration of SMCs and further accumulation of inflammatory cells, leading to neointima formation ${ }^{54)}$. These inflammatory cells and SMCs are thought to migrate from the bloodstream and proliferate within the lesion. Atherosclerosis is thought to progress "inside-out" from the endothelium to the adventitia. A recent study has elucidated that atherosclerotic plaque growth is accompanied by a network of microvessels, called the vasa vasorum, extend- 
ing from the adventitia into the base of the plaque. The vasa vasorum supplies oxygen and nutrients to the outer layers of the arterial wall ${ }^{55,56)}$. It is believed that inflammatory cytokines and chemotactic factors stimulate SMC migration via the vasa vasorum. Thus, the concept that the atherosclerotic process extends not only inside-out but also "outside-in" is widely believed. SMC migration contributes to plaque buildup.

It has been shown that n-3 PUFAs block SMC proliferation and migration ${ }^{57)}$. We demonstrated that EPA treatment reduced SMC proliferation and atherosclerotic lesion development in $A p o e^{-/-}$mice fed a Western-type diet ${ }^{58)}$. An abundant development of the vasa vasorum around the coronary artery of a hypercholesterolemic pig showed an enhanced expression of the vascular endothelial growth factor (VEGF). Statins reportedly attenuate the neovascularization of the vasa vasorum via VEGF inhibition ${ }^{59,60)}$. EPA attenuates the tube formation of endothelial cells via its inhibitory effect on cellular proliferation ${ }^{61)}$. Thus, $\mathrm{n}-3$ PUFAs could inhibit the neovascularization of the vasa vasorum and reduce the migration of SMCs and inflammatory cells, leading to the suppression of plaque development ${ }^{61)}$.

\section{5) Plaque Vulnerability Leading to Plaque Rupture}

Ultimately, inflammatory mediators can inhibit collagen synthesis and evoke collagenase expression by foam cells within the intimal lesion. Macrophages internalize cholesterol through their scavenger receptors and produce inflammatory cytokines and collagenases, including matrix metalloproteinases (MMPs) ${ }^{62)}$. MMPs cause thinning of the fibrous cap and render it weak and susceptible to rupture at the plaque shoulder, leading to plaque rupture. The subendothelial tissue factor is then uncovered, and the tissue factor induced by the inflammatory signaling triggers thrombus formation, resulting in $\mathrm{ACS}^{63)}$.

Nozue et al. have shown that decrease in serum n-3 to n-6 polyunsaturated fatty acid ratio is associated with the progression of coronary atherosclerosis, which was evaluated using virtual histology intravascular ultrasound in statin-treated patients with $\mathrm{CAD}^{64)}$. We showed that EPA treatment in addition to statin treatment reduced lipid volume in coronary plaques, which was evaluated by integrated backscatter intravascular ultrasound, and decreased inflammatory cytokines including pentraxin 3 and MCP-1 ${ }^{65)}$.

A histological study showed that carotid atherosclerotic plaques from patients treated with fish oil were less heavily infiltrated with macrophages than in those in the placebo group. Moreover, plaques from patients treated with fish oil were more likely to be fibrous-cap atheromas and display fewer signs of inflammation than those treated with placebo, indicating that fish oil increases plaque stability ${ }^{66)}$.

Decreased EPA/AA ratios were identified in young Japanese subjects ${ }^{67)}$. Decreased EPA/AA and DHA/ $\mathrm{AA}$ ratios are known risk factors for early-onset ACS, suggesting that reduced EPA/AA and DHA/AA ratios may represent targets for preventing ACS in young Japanese people ${ }^{68)}$. There is a high prevalence of coronary atherosclerosis in asymptomatic young people, suggesting that $n-3$ PUFA intake from a young age might be needed to prevent future ACS ${ }^{69,70)}$.

Taken together, n-3 PUFAs attenuate all the above features of atherogenesis similar to statins (Fig. 4).

\section{Other Preferable Effects of n-3 PUFAs for Preventing CVD}

\section{1) n-3 PUFAs Prevent Cardiac Remodeling and Heart Failure}

CVD events eventually lead to heart failure. The OMEGA-REMODEL randomized clinical trial has shown that high-dose n-3 PUFA treatment in patients with acute myocardial infarction was associated with reduced adverse left ventricular remodeling, non-infarct myocardial fibrosis, evaluated by cardiac magnetic resonance and serum biomarkers of systemic inflammation beyond the current guideline-based standard of care $^{71)}$. GISSI HF investigated whether n-3 PUFA could improve morbidity and mortality rates in a large population of patients with symptomatic heart failure of any cause ${ }^{72}$. The findings of this study indicated that n-3 PUFAs have preferable effects on cardiac remodeling and heart failure.

\section{2) n-3 PUFAs Attenuates Ventricular Arrhythmia}

The onset of CVD and/or heart failure induces fatal ventricular arrhythmia ${ }^{73)}$. Epidemiological studies have shown that n-3 PUFA intake reduced sudden cardiac death and ventricular arrhythmias and showed an inverse relationship between n-3 PUFA concentration and the prevalence of sudden cardiac death ${ }^{74)}$. n-3 PUFAs may have profound effects on the trafficking of ion channels through subcellular compartments and within lipid rafts. In addition, n-3 PUFAs can inhibit the $\mathrm{Na}^{+}$current and several different $\mathrm{K}^{+}$currents in a dose-dependent manner and can modulate or attenuate $\mathrm{Ca}^{2+}$ influx into cardiomyocytes and sodiumcalcium exchanger system. These mechanisms may contribute to the anti-arrhythmic effects of n-3 PUFAs that lead to the prevention of sudden cardiac death.

We reported that low serum EPA and DHA levels are risk factors for cardiogenic syncope in patients with Brugada syndrome ${ }^{75)}$. Although the etiology of 


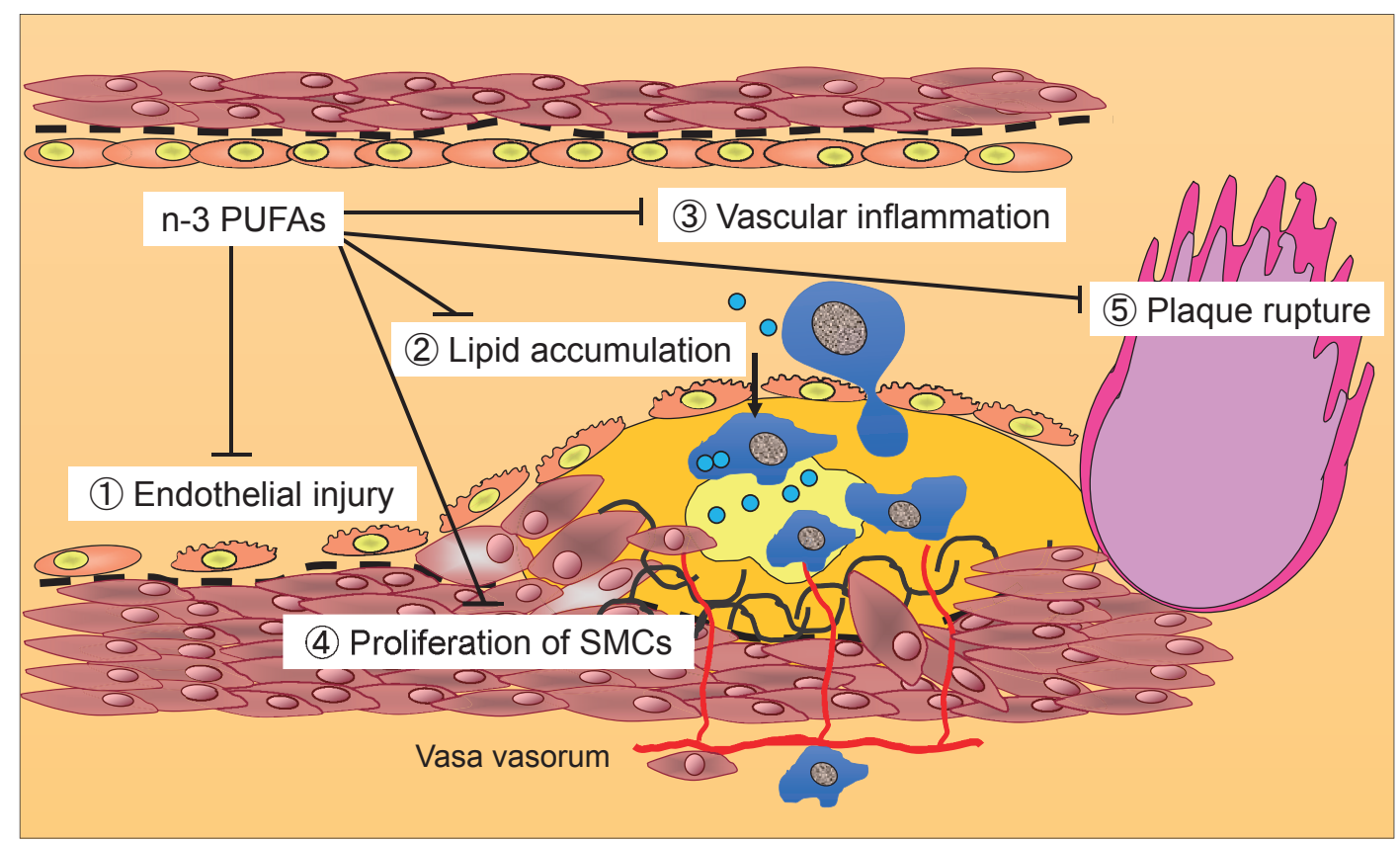

Fig.4. n-3 PUFAs attenuate all components of atherosclerosis.

Brugada syndrome is multifactorial and involves genetic, environmental, and hormonal components, n-3 PUFAs can modulate cardiac ion channels, resulting in the prevention of ventricular arrhythmia.

\section{3) n-3 PUFAs Improve Cognitive Function}

Cognitive function or a depressed mood are known risk factors for cardiovascular events ${ }^{76,77)}$. n-3 PUFAs play important roles in cardiovascular protection and neuroprotection. We showed that serum EPA level is associated with cognitive function in patients with $\mathrm{CAD}$, independent of cardiac function ${ }^{78)}$. A doubleblind, randomized controlled trial has demonstrated that n-3 PUFA supplementation is associated with improved cognitive function compared with control group with placebo in patients with mild cognitive impairment ${ }^{79)}$. In addition, several studies have reported that the negligible effect of n-3 PUFAs in patients with Alzheimer's disease may be through an increasing clearance of amyloid- $\beta$ peptide, neurotrophic and neuroprotective factors, and anti-inflammatory effects ${ }^{80}$. Thus, a reduced level of n-3 PUFA is a risk factor for cognitive dysfunction, and n-3 PUFAs intake could improve cognitive function, decreasing the risk of cardiovascular events ${ }^{78,81}$.

\section{4) n-3 PUFAs Improve Exercise Capacity}

Reduced exercise capacity is also a known risk factor for cardiovascular events ${ }^{82}$. n-3 PUFAs could improve exercise capacity in patients with $\mathrm{CVD}^{83,84)}$.
Although the mechanisms by which n-3 PUFAs improve exercise capacity has not been fully elucidated, it is known that the incorporation of n-3 PUFAs into myocardial and skeletal muscle membranes leads to the modification of skeletal muscle function and increases insulin sensitivity and that of n-3 PUFAs into erythrocytes leads to the modification of erythrocyte rheology, which might be involved in n-3 PUFAinduced increases in exercise capacity ${ }^{84)}$.

\section{Who Should Take n-3 PUFAs? Patients with CVD and Heart Failure}

The effects of $n-3$ PUFAs on CVD prevention seems modest compared with those of statins, and the best candidates to receive the benefits of n-3 PUFAs on CVD prevention are yet to be identified. A recent recommendation from the American Heart Association suggests that $n-3$ PUFA supplementation in patients with CVD for secondary prevention and those with heart failure is reasonable from randomized control trials ${ }^{85)}$. However, further large-scale clinical trials are needed to identify the best candidates for n-3 PUFA supplementation to prevent CVD because of a lack of evidence.

\section{Conclusions}

A growing body of evidence suggests that $n-3$ PUFAs have beneficial effects for preventing CVD in 
this era of statins. n-3 PUFAs suppress atherosclerosis via pleiotropic effects in addition to modifying lipid profiles. Thus, n-3 PUFA intake is recommended to prevent CVD, particularly in patients with multiple cardiovascular risk factors.

\section{Conflict of Interest}

M. Sata received research funding from TanabeMitsubishi, Takeda, Astellas, Byer Healthcare, DaiichiSankyo, MSD, and Ono and lecture fees from Astellas, Boehringer Ingelheim, Byer Healthcare, Mochida, Takeda, Tanabe-Mitsubishi, Novartis, AstraZeneca, MSD, and Shionogi. The Department of Cardio-Diabetes Medicine, Tokushima University Graduate School is supported in part by unrestricted research grants from Actelion, Boehringer Ingelheim, Kowa, and Tanabe-Mitsubishi. The others declare that they have no conflicts of interest to disclose.

\section{References}

1) Libby P: The forgotten majority: unfinished business in cardiovascular risk reduction. J Am Coll Cardiol 2005, 46: $1225-1228$

2) Hara M, Sakata Y, Nakatani D, Suna S, Usami M, Matsumoto S, Hamasaki T, Doi Y, Nishino M, Sato H, Kitamura T, Nanto S, Hori M, Komuro I, Osaka Acute Coronary Insufficiency Study I: Low levels of serum n-3 polyunsaturated fatty acids are associated with worse heart failure-free survival in patients after acute myocardial infarction. Circ J 2013, 77: 153-162

3) Yokoyama M, Origasa H, Matsuzaki M, Matsuzawa Y, Saito Y, Ishikawa Y, Oikawa S, Sasaki J, Hishida H, Itakura H, Kita T, Kitabatake A, Nakaya N, Sakata T, Shimada K, Shirato K, Japan EPAlisI: Effects of eicosapentaenoic acid on major coronary events in hypercholesterolaemic patients (JELIS): a randomised open-label, blinded endpoint analysis. Lancet 2007, 369: 1090-1098

4) Das UN: Essential fatty acids and their metabolites could function as endogenous HMG-CoA reductase and ACE enzyme inhibitors, anti-arrhythmic, anti-hypertensive, antiatherosclerotic, anti-inflammatory, cytoprotective, and cardioprotective molecules. Lipids Health Dis 2008, 7: 37

5) Goyens PL, Spilker ME, Zock PL, Katan MB, Mensink RP: Compartmental modeling to quantify alpha-linolenic acid conversion after longer term intake of multiple tracer boluses. J Lipid Res 2005, 46: 1474-1483

6) Hussein N, Ah-Sing E, Wilkinson P, Leach C, Griffin BA, Millward DJ: Long-chain conversion of [13C]linoleic acid and alpha-linolenic acid in response to marked changes in their dietary intake in men. J Lipid Res 2005, 46: 269280

7) Swanson D, Block R, Mousa SA: Omega-3 fatty acids EPA and DHA: health benefits throughout life. Adv Nutr 2012, 3: $1-7$

8) Simopoulos AP: Essential fatty acids in health and chronic disease. Am J Clin Nutr 1999, 70: 560S-569S
9) Ross R: Atherosclerosis--an inflammatory disease. N Engl J Med 1999, 340: 115-126

10) Grundy SM: HMG-CoA reductase inhibitors for treatment of hypercholesterolemia. N Engl J Med 1988, 319: 24-33

11) Reith C, Armitage J: Management of residual risk after statin therapy. Atherosclerosis 2016, 245: 161-170

12) Yagi S, Aihara K, Ikeda Y, Akaike M, Sata M, Matsumoto T: Effects of statins on cardiorenal syndrome. Int J Vasc Med 2012, 2012: 162545

13) Yagi S, Aihara K, Ikeda Y, Sumitomo Y, Yoshida S, Ise T, Iwase T, Ishikawa K, Azuma H, Akaike M, Matsumoto T: Pitavastatin, an HMG-CoA reductase inhibitor, exerts eNOS-independent protective actions against angiotensin II induced cardiovascular remodeling and renal insufficiency. Circ Res 2008, 102: 68-76

14) Yagi S, Akaike M, Aihara K, Ishikawa K, Iwase T, Ikeda $Y$, Soeki T, Yoshida S, Sumitomo-Ueda Y, Matsumoto T, Sata M: Endothelial nitric oxide synthase-independent protective action of statin against angiotensin II-induced atrial remodeling via reduced oxidant injury. Hypertension 2010, 55: 918-923

15) Jula A, Marniemi J, Ronnemaa T, Virtanen A, Huupponen R: Effects of diet and simvastatin on fatty acid composition in hypercholesterolemic men: a randomized controlled trial. Arterioscler Thromb Vasc Biol 2005, 25: $1952-$ 1959

16) Nozue T, Yamamoto $S$, Tohyama $S$, Fukui K, Umezawa $S$, Onishi Y, Kunishima T, Sato A, Nozato T, Miyake S, Takeyama Y, Morino Y, Yamauchi T, Muramatsu T, Hibi K, Michishita I: Effects of statins on serum n-3 to n-6 polyunsaturated fatty acid ratios in patients with coronary artery disease. J Cardiovasc Pharmacol Ther 2013, 18: 320-326

17) Kurisu S, Ishibashi K, Kato Y, Mitsuba N, Dohi Y, Nishioka K, Kihara Y: Effects of lipid-lowering therapy with strong statin on serum polyunsaturated fatty acid levels in patients with coronary artery disease. Heart Vessels 2013, 28: 34-38

18) Harris JI, Hibbeln JR, Mackey RH, Muldoon MF: Statin treatment alters serum n-3 and n-6 fatty acids in hypercholesterolemic patients. Prostaglandins Leukot Essent Fatty Acids 2004, 71: 263-269

19) Nakamura N, Hamazaki T, Jokaji H, Minami S, Kobayashi M: Effect of HMG-CoA reductase inhibitors on plasma polyunsaturated fatty acid concentrations in patients with hyperlipidemia. Int J Clin Lab Res 1998, 28: 192-195

20) Dyerberg J, Bang HO, Stoffersen E, Moncada S, Vane JR: Eicosapentaenoic acid and prevention of thrombosis and atherosclerosis? Lancet 1978, 2: 117-119

21) Yamada T, Strong JP, Ishii T, Ueno T, Koyama M, Wagayama H, Shimizu A, Sakai T, Malcom GT, Guzman MA: Atherosclerosis and omega-3 fatty acids in the populations of a fishing village and a farming village in Japan. Atherosclerosis 2000, 153: 469-481

22) Iso H, Kobayashi M, Ishihara J, Sasaki S, Okada K, Kita Y, Kokubo Y, Tsugane S, Group JS: Intake of fish and n3 fatty acids and risk of coronary heart disease among Japanese: the Japan Public Health Center-Based (JPHC) Study Cohort I. Circulation 2006, 113: 195-202

23) Kromhout D, Bosschieter EB, de Lezenne Coulander C: The inverse relation between fish consumption and 20-year 
mortality from coronary heart disease. N Engl J Med 1985, 312: 1205-1209

24) Wang C, Harris WS, Chung M, Lichtenstein AH, Balk EM, Kupelnick B, Jordan HS, Lau J: n-3 Fatty acids from fish or fish-oil supplements, but not alpha-linolenic acid, benefit cardiovascular disease outcomes in primary- and secondary-prevention studies: a systematic review. Am J Clin Nutr 2006, 84: 5-17

25) He K, Song Y, Daviglus ML, Liu K, Van Horn L, Dyer AR, Greenland P: Accumulated evidence on fish consumption and coronary heart disease mortality: a meta-analysis of cohort studies. Circulation 2004, 109: 2705-2711

26) Burr ML, Fehily AM, Gilbert JF, Rogers S, Holliday RM, Sweetnam PM, Elwood PC, Deadman NM: Effects of changes in fat, fish, and fibre intakes on death and myocardial reinfarction: diet and reinfarction trial (DART). Lancet 1989, 2: 757-761

27) Dietary supplementation with n-3 polyunsaturated fatty acids and vitamin $\mathrm{E}$ after myocardial infarction: results of the GISSI-Prevenzione trial. Gruppo Italiano per lo Studio della Sopravvivenza nell'Infarto miocardico. Lancet 1999, 354: 447-455

28) Studer M, Briel M, Leimenstoll B, Glass TR, Bucher HC: Effect of different antilipidemic agents and diets on mortality: a systematic review. Arch Intern Med 2005, 165: 725-730

29) Erkkila AT, Matthan NR, Herrington DM, Lichtenstein $\mathrm{AH}$ : Higher plasma docosahexaenoic acid is associated with reduced progression of coronary atherosclerosis in women with CAD. J Lipid Res 2006, 47: 2814-2819

30) Takashima A, Fukuda D, Tanaka K, Higashikuni Y, Hirata Y, Nishimoto S, Yagi S, Yamada H, Soeki T, Wakatsuki T, Taketani Y, Shimabukuro M, Sata M: Combination of $\mathrm{n}-3$ polyunsaturated fatty acids reduces atherogenesis in apolipoprotein E-deficient mice by inhibiting macrophage activation. Atherosclerosis 2016, 254: 142-150

31) Arterburn LM, Hall EB, Oken H: Distribution, interconversion, and dose response of $\mathrm{n}-3$ fatty acids in humans. Am J Clin Nutr 2006, 83: 1467S-1476S

32) Mori-Kawabe M, Tsushima $H$, Fujimoto $S$, Tada T, Ito J: Role of Rho/Rho-kinase and NO/cGMP signaling pathways in vascular function prior to atherosclerosis. J Atheroscler Thromb 2009, 16: 722-732

33) Wang Q, Liang X, Wang L, Lu X, Huang J, Cao J, Li H, Gu D: Effect of omega-3 fatty acids supplementation on endothelial function: a meta-analysis of randomized controlled trials. Atherosclerosis 2012, 221: 536-543

34) Yagi S, Aihara KI, Fukuda D, Takashima A, Hara T, Hotchi J, Ise T, Yamaguchi K, Tobiume T, Iwase T, Yamada H, Soeki T, Wakatsuki T, Shimabukuro M, Akaike M, Sata M: Effects of Docosahexaenoic Acid on the Endothelial Function in Patients with Coronary Artery Disease. J Atheroscler Thromb 2015; 22: 447-454

35) Mori TA, Watts GF, Burke V, Hilme E, Puddey IB, Beilin LJ: Differential effects of eicosapentaenoic acid and docosahexaenoic acid on vascular reactivity of the forearm microcirculation in hyperlipidemic, overweight men. Circulation 2000, 102: 1264-1269

36) Kelley DS, Adkins Y: Similarities and differences between the effects of EPA and DHA on markers of atherosclerosis in human subjects. Proc Nutr Soc 2012, 71: 322-331
37) Chen J, Shearer GC, Chen Q, Healy CL, Beyer AJ, Nareddy VB, Gerdes AM, Harris WS, O'Connell TD, Wang D: Omega-3 fatty acids prevent pressure overload-induced cardiac fibrosis through activation of cyclic GMP/protein kinase $\mathrm{G}$ signaling in cardiac fibroblasts. Circulation 2011, 123: 584-593

38) Dimitrow PP, Jawien M: Pleiotropic, cardioprotective effects of omega-3 polyunsaturated fatty acids. Mini Rev Med Chem 2009, 9: 1030-1039

39) Omura M, Kobayashi S, Mizukami Y, Mogami K, Todoroki-Ikeda N, Miyake T, Matsuzaki M: Eicosapentaenoic acid (EPA) induces $\mathrm{Ca}(2+)$-independent activation and translocation of endothelial nitric oxide synthase and endothelium-dependent vasorelaxation. FEBS Lett 2001, 487: 361-366

40) Ishida T, Naoe S, Nakakuki M, Kawano H, Imada K: Eicosapentaenoic Acid Prevents Saturated Fatty Acid-Induced Vascular Endothelial Dysfunction: Involvement of LongChain Acyl-CoA Synthetase. J Atheroscler Thromb 2015, 22: $1172-1185$

41) Nordestgaard BG, Varbo A: Triglycerides and cardiovascular disease. Lancet 2014, 384: 626-635

42) Mori TA: Dietary n-3 PUFA and CVD: a review of the evidence. Proc Nutr Soc 2014, 73: 57-64

43) Hokanson JE, Austin MA: Plasma triglyceride level is a risk factor for cardiovascular disease independent of highdensity lipoprotein cholesterol level: a meta-analysis of population-based prospective studies. J Cardiovasc Risk 1996, 3: 213-219

44) Harris WS, Miller M, Tighe AP, Davidson MH, Schaefer EJ: Omega-3 fatty acids and coronary heart disease risk: clinical and mechanistic perspectives. Atherosclerosis 2008, 197: 12-24

45) Satoh N, Shimatsu A, Kotani K, Sakane N, Yamada K, Suganami T, Kuzuya H, Ogawa Y: Purified eicosapentaenoic acid reduces small dense LDL, remnant lipoprotein particles, and C-reactive protein in metabolic syndrome. Diabetes Care 2007, 30: 144-146

46) Calder PC: The role of marine omega-3 (n-3) fatty acids in inflammatory processes, atherosclerosis and plaque stability. Mol Nutr Food Res 2012, 56: 1073-1080

47) Robinson JG, Stone NJ: Antiatherosclerotic and antithrombotic effects of omega-3 fatty acids. Am J Cardiol 2006, 98: 39i-49i

48) De Caterina R, Liao JK, Libby P: Fatty acid modulation of endothelial activation. Am J Clin Nutr 2000, 71: 213S$223 \mathrm{~S}$

49) Shimizu T, Iwamoto $T$, Itou $S$, Iwata $N$, Endo T, Takasaki M: Effect of ethyl icosapentaenoate (EPA) on the concentration of tumor necrosis factor (TNF) and interleukin-1 (IL-1) in the carotid artery of cuff-sheathed rabbit models. J Atheroscler Thromb 2001, 8: 45-49

50) Calder PC: $n-3$ polyunsaturated fatty acids, inflammation, and inflammatory diseases. Am J Clin Nutr 2006, 83: 1505S-1519S

51) Nishimoto $S$, Fukuda D, Higashikuni $Y$, Tanaka K, Hirata Y, Murata C, Kim-Kaneyama JR, Sato F, Bando M, Yagi S, Soeki T, Hayashi T, Imoto I, Sakaue H, Shimabukuro M, Sata M: Obesity-induced DNA released from adipocytes stimulates chronic adipose tissue inflammation and insulin resistance. Sci Adv 2016, 2: e1501332 
52) Tall AR, Yvan-Charvet L: Cholesterol, inflammation and innate immunity. Nat Rev Immunol 2015, 15: 104-116

53) Ciesielska A, Kwiatkowska K: Modification of pro-inflammatory signaling by dietary components: The plasma membrane as a target. Bioessays 2015, 37: 789-801

54) Katsuda S, Okada Y: Vascular smooth muscle cell migration and extracellular matrix. J Atheroscler Thromb 1994, 1 Suppl 1: S34-38

55) Barger AC, Beeuwkes R, 3rd, Lainey LL, Silverman KJ: Hypothesis: vasa vasorum and neovascularization of human coronary arteries. A possible role in the pathophysiology of atherosclerosis. N Engl J Med 1984, 310: 175-177

56) Tanaka K, Sata M: Visualization of the human coronary vasa vasorum in vivo. Circ J 2015, 79: 1211-1212

57) Pakala R, Pakala R, Sheng WL, Benedict CR: Eicosapentaenoic acid and docosahexaenoic acid block serotonininduced smooth muscle cell proliferation. Arterioscler Thromb Vasc Biol 1999, 19: 2316-2322

58) Matsumoto M, Sata M, Fukuda D, Tanaka K, Soma M, Hirata Y, Nagai R: Orally administered eicosapentaenoic acid reduces and stabilizes atherosclerotic lesions in ApoEdeficient mice. Atherosclerosis 2008, 197: 524-533

59) Wilson SH, Herrmann J, Lerman LO, Holmes DR, Jr., Napoli C, Ritman EL, Lerman A: Simvastatin preserves the structure of coronary adventitial vasa vasorum in experimental hypercholesterolemia independent of lipid lowering. Circulation 2002, 105: 415-418

60) Sato I, Ma L, Ikeda M, Morita I, Murota S: Simvastatin, a potent HMG-CoA reductase inhibitor, inhibits the proliferation of human and bovine endothelial cells in vitro. J Atheroscler Thromb 1998, 4: 102-106

61) Kanayasu T, Morita I, Nakao-Hayashi J, Asuwa N, Fujisawa $\mathrm{C}$, Ishii T, Ito H, Murota S: Eicosapentaenoic acid inhibits tube formation of vascular endothelial cells in vitro. Lipids 1991, 26: 271-276

62) Okamoto Y, Satomura K, Ohsuzu F, Nakamura H, Takeuchi K, Yoshioka M: Expression of matrix metalloproteinase 3 in experimental atherosclerotic plaques. J Atheroscler Thromb 2001, 8: 50-54

63) Libby P, Ridker PM, Maseri A: Inflammation and atherosclerosis. Circulation 2002, 105: 1135-1143

64) Nozue T, Yamamoto $S$, Tohyama $S$, Fukui $K$, Umezawa $S$, Onishi Y, Kunishima T, Sato A, Nozato T, Miyake S, Takeyama Y, Morino Y, Yamauchi T, Muramatsu T, Hibi K, Terashima M, Michishita I: Effects of serum n-3 to n-6 polyunsaturated fatty acids ratios on coronary atherosclerosis in statin-treated patients with coronary artery disease. Am J Cardiol 2013, 111: 6-11

65) Niki T, Wakatsuki T, Yamaguchi K, Taketani Y, Oeduka H, Kusunose K, Ise T, Iwase T, Yamada H, Soeki T, Sata M: Effects of the Addition of Eicosapentaenoic Acid to Strong Statin Therapy on Inflammatory Cytokines and Coronary Plaque Components Assessed by Integrated Backscatter Intravascular Ultrasound. Circ J 2016, 80: 450-460

66) Thies F, Garry JM, Yaqoob P, Rerkasem K, Williams J, Shearman CP, Gallagher PJ, Calder PC, Grimble RF: Association of $n-3$ polyunsaturated fatty acids with stability of atherosclerotic plaques: a randomised controlled trial. Lancet 2003, 361: 477-485

67) Yanagisawa N, Shimada K, Miyazaki T, Kume A, Kitamura Y, Ichikawa R, Ohmura H, Kiyanagi T, Hiki M,
Fukao K, Sumiyoshi K, Hirose K, Matsumori R, Takizawa H, Fujii K, Mokuno H, Inoue N, Daida H: Polyunsaturated fatty acid levels of serum and red blood cells in apparently healthy Japanese subjects living in an urban area. J Atheroscler Thromb 2010, 17: 285-294

68) Yagi S, Aihara K, Fukuda D, Takashima A, Bando M, Hara T, Nishimoto S, Ise T, Kusunose K, Yamaguchi K, Tobiume T, Iwase T, Yamada H, Soeki T, Wakatsuki T, Shimabukuro M, Akaike M, Sata M: Reduced ratio of eicosapentaenoic acid and docosahexaenoic acid to arachidonic acid is associated with early onset of acute coronary syndrome. Nutr J 2015, 14: 111

69) Kisanuki A, Asada Y, Sato Y, Marutsuka K, Takeda K, Sumiyoshi A: Coronary atherosclerosis in youths in Kyushu Island, Japan: histological findings and stenosis. J Atheroscler Thromb 2000, 6: 55-59

70) Tuzcu EM, Kapadia SR, Tutar E, Ziada KM, Hobbs RE, McCarthy PM, Young JB, Nissen SE: High prevalence of coronary atherosclerosis in asymptomatic teenagers and young adults: evidence from intravascular ultrasound. Circulation 2001, 103: 2705-2710

71) Heydari B, Abdullah S, Pottala JV, Shah R, Abbasi S, Mandry D, Francis SA, Lumish H, Ghoshhajra BB, Hoffmann U, Appelbaum E, Feng JH, Blankstein R, Steigner M, McConnell JP, Harris W, Antman EM, Jerosch-Herold M, Kwong RY: Effect of Omega-3 Acid Ethyl Esters on Left Ventricular Remodeling After Acute Myocardial Infarction: The OMEGA-REMODEL Randomized Clinical Trial. Circulation 2016, 134: 378-391

72) Tavazzi L, Maggioni AP, Marchioli R, Barlera S, Franzosi MG, Latini R, Lucci D, Nicolosi GL, Porcu M, Tognoni G, Gissi HFI: Effect of $n-3$ polyunsaturated fatty acids in patients with chronic heart failure (the GISSI-HF trial): a randomised, double-blind, placebo-controlled trial. Lancet 2008, 372: 1223-1230

73) Yagi S, Tsuda E, Shimizu W, Kurita T, Seguchi O, Nonogi $\mathrm{H}$, Kamakura S: Two adults requiring implantable defibrillators because of ventricular tachycardia and left ventricular dysfunction caused by presumed Kawasaki disease. Circ J 2005, 69: 870-874

74) London B, Albert C, Anderson ME, Giles WR, Van Wagoner DR, Balk E, Billman GE, Chung M, Lands W, Leaf A, McAnulty J, Martens JR, Costello RB, Lathrop DA: Omega-3 fatty acids and cardiac arrhythmias: prior studies and recommendations for future research: a report from the National Heart, Lung, and Blood Institute and Office Of Dietary Supplements Omega-3 Fatty Acids and their Role in Cardiac Arrhythmogenesis Workshop. Circulation 2007, 116: e320-335

75) Yagi S, Soeki T, Aihara K, Fukuda D, Ise T, Kadota M, Bando S, Matsuura T, Tobiume T, Yamaguchi K, Kusunose K, Yamada H, Wakatsuki T, Shimabukuro M, Akaike $\mathrm{M}$, and Sata M: Low serum levels of eicosapentaenoic acid and docosahexaenoic acid are risk factors for cardiogenic syncope in patients with Brugada syndrome. Int Heart J (in press)

76) Yagi S, Akaike M, Aihara K, Iwase T, Yoshida S, Sumitomo-Ueda Y, Ikeda Y, Ishikawa K, Matsumoto T, Sata M: High plasma aldosterone concentration is a novel risk factor of cognitive impairment in patients with hypertension. Hypertens Res 2011, 34: 74-78 
77) Yagi S, Akaike M, Ise T, Ueda Y, Iwase T, Sata M: Reninangiotensin-aldosterone system has a pivotal role in cognitive impairment. Hypertens Res 2013, 36: 753-758

78) Yagi S, Hara T, Ueno R, Aihara KI, Fukuda D, Takashima A, Hotchi J, Ise T, Yamaguchi K, Tobiume T, Iwase T, Yamada H, Soeki T, Wakatsuki T, Shimabukuro M, Akaike M, Sata M: Serum concentration of eicosapentaenoic acid is associated with cognitive function in patients with coronary artery disease. Nutr J 2014, 13: 112

79) Bo Y, Zhang X, Wang Y, You J, Cui H, Zhu Y, Pang W, Liu W, Jiang Y, Lu Q: The n-3 Polyunsaturated Fatty Acids Supplementation Improved the Cognitive Function in the Chinese Elderly with Mild Cognitive Impairment: A Double-Blind Randomized Controlled Trial. Nutrients 2017, 9

80) Yanai H: Effects of N-3 Polyunsaturated Fatty Acids on Dementia. J Clin Med Res 2017, 9: 1-9

81) Appleton KM, Hayward RC, Gunnell D, Peters TJ, Rogers PJ, Kessler D, Ness AR: Effects of n-3 long-chain polyunsaturated fatty acids on depressed mood: systematic review of published trials. Am J Clin Nutr 2006, 84: 13081316
82) Powell KE, Thompson PD, Caspersen CJ, Kendrick JS: Physical activity and the incidence of coronary heart disease. Annu Rev Public Health 1987, 8: 253-287

83) Moyers B, Farzaneh-Far R, Harris WS, Garg S, Na B, Whooley MA: Relation of whole blood n-3 fatty acid levels to exercise parameters in patients with stable coronary artery disease (from the heart and soul study). Am J Cardiol 2011, 107: 1149-1154

84) Da Boit M, Hunter AM, Gray SR: Fit with good fat? The role of $\mathrm{n}-3$ polyunsaturated fatty acids on exercise performance. Metabolism 2017, 66: 45-54

85) Siscovick DS, Barringer TA, Fretts AM, Wu JH, Lichtenstein AH, Costello RB, Kris-Etherton PM, Jacobson TA, Engler MB, Alger HM, Appel LJ, Mozaffarian D, American Heart Association Nutrition Committee of the Council on L, Cardiometabolic H, Council on E, Prevention, Council on Cardiovascular Disease in the Y, Council on C, Stroke N, Council on Clinical C: Omega-3 Polyunsaturated Fatty Acid (Fish Oil) Supplementation and the Prevention of Clinical Cardiovascular Disease: A Science Advisory From the American Heart Association. Circulation 2017, 135: e867-e884 